\title{
ON GENERALIZED MAX-LINEAR MODELS AND THEIR STATISTICAL INTERPOLATION
}

\author{
MICHAEL FALK, ${ }^{* * *}$ \\ MARTIN HOFMANN **** AND \\ MAXIMILIAN ZOTT, ${ }^{* * * * *}$ University of Wurzburg
}

\begin{abstract}
We propose a method to generate a max-stable process in $C[0,1]$ from a max-stable random vector in $\mathbb{R}^{d}$ by generalizing the max-linear model established by Wang and Stoev (2011). For this purpose, an interpolation technique that preserves max-stability is proposed. It turns out that if the random vector follows some finite-dimensional distribution of some initial max-stable process, the approximating processes converge uniformly to the original process and the pointwise mean-squared error can be represented in a closed form. The obtained results carry over to the case of generalized Pareto processes. The introduced method enables the reconstruction of the initial process only from a finite set of observation points and, thus, a reasonable prediction of max-stable processes in space becomes possible. A possible extension to arbitrary dimensions is outlined.
\end{abstract}

Keywords: Multivariate extreme value distribution; multivariate generalized Pareto distribution; max-stable process; generalized Pareto process; $D$-norm; max-linear model; prediction of max-stable process; prediction of generalized Pareto process

2010 Mathematics Subject Classification: Primary 60G70

\section{Introduction and prelimiaries}

\subsection{Introduction}

A max-stable process (MSP) $\xi=\left(\xi_{t}\right)_{t \in K}$ with sample paths in

$$
C(K):=\left\{g \in \mathbb{R}^{K}: g \text { is continuous }\right\}
$$

with a compact set $K \subset \mathbb{R}$ has the characteristic property that there are continuous functions $a_{n}>0, b_{n} \in C(K)$ for every $n \in \mathbb{N}$ such that

$$
\frac{\max _{1 \leq i \leq n}\left(\xi^{(i)}-b_{n}\right)}{a_{n}}=\left(\frac{\max _{1 \leq i \leq n}\left(\xi_{t}(i)-b_{n}(t)\right)}{a_{n}(t)}\right)_{t \in K} \stackrel{\mathrm{D}}{=} \xi,
$$

where $\xi^{(i)}, i=1, \ldots, n$, are independent copies of $\boldsymbol{\xi}$ and $\stackrel{\text { D }}{=}$ ' denotes equality in distribution. It is well known (see, e.g. De Haan and Ferreira (2006)), that MSPs are the only possible limit processes of linearly standardized maxima of independent and identically distributed processes.

Received 5 June 2013; revision received 5 June 2014.

* Postal address: Institute of Mathematics, University of Wurzburg, Emil-Fischer-Str. 30, 97074 Würzburg, Germany.

** Email address: michael.falk@uni-wuerzburg.de

*** Email address: hofmann.martin@mathematik.uni-wuerzburg.de

**** Email address: maximilian.zott@uni-wuerzburg.de 
This is in complete accordance to the well-established finite-dimensional case of extreme value analysis; see, e.g. Falk et al. (2011) and De Haan and Ferreira (2006).

The theory of continuous MSPs is essentially based on the early work of De Haan (1984), Giné et al. (1990), and De Haan and Lin (2001), followed by recent findings with the focus on different aspects within the theory; see, e.g. Hult and Lindskog (2005), (2006), Stoev and Taqqu (2005), Davis and Mikosch (2008), Kabluchko (2009), Wang and Stoev (2010), and Aulbach et al. (2013).

Moreover, the class of excursion stable generalized Pareto processes, which is closely related to the class of MSPs, was examined in Buishand et al. (2008) and Aulbach et al. (2013); the most recent advances on that issue can be found in Ferreira and De Haan (2014).

There is a very crucial problem in the theory of stochastic processes that concerns its relevance in practice: as (continuous) processes as a whole cannot be measured exactly, the question is how to construct those processes (with some characteristic stochastic behavior such as maxstability) from a finite set of observations. As an example, one can think of data from a finite set of measuring stations metering the sea level along a coast and one is interested in predicting the sea level between those measuring stations. This means the 'prediction' of a stochastic process in space, not in time.

In the case of MSPs (or fields, if the domain has more than one dimension), there are (partial) answers on the arising questions in Wang and Stoev (2011) and Dombry et al. (2013) based on conditional sampling. Different from that, our approach is conditionally deterministic. We apply an interpolation technique that preserves the max-stability of some initial random vector (RV).

In this paper we pick up the so-called 'max-linear model' introduced in Wang and Stoev (2011): for arbitrary nonnegative continuous functions $g_{0}, \ldots, g_{d}$ satisfying (1) below, we obtain an MSP $\boldsymbol{\eta}=\left(\eta_{t}\right)_{t \in[0,1]}$ by setting

$$
\eta_{s}=\max _{j=0, \ldots, d} \frac{X_{j}}{g_{j}(s)}, \quad s \in[0,1],
$$

where $\boldsymbol{X}=\left(X_{0}, \ldots, X_{d}\right)$ is a max-stable $\mathrm{RV}$ with independent components. The obvious restriction of this model is the required independence of the margins of $\boldsymbol{X}$, which results in the fact that $\boldsymbol{\eta}$ always has a discrete spectral measure (the spectral measure in our setup is described below and represented by the distribution of the generator process $\boldsymbol{Z}$; see Aulbach et al. (2013)).

In Section 2 we generalize this model by allowing arbitrary dependence structures of the margins of the max-stable RV $\boldsymbol{X}$. This immediately leads to the main issue of this paper, namely the reconstruction of an MSP which is observed only through a finite set of indices. In Section 3 we show that if the RV is some finite-dimensional projection of some initial MSP, the process resulting from the construction in Section 2 converges uniformly to the original process as the grid of indices becomes finer. Moreover, the mean-squared errors between the predictive and the original process is computed at a fixed index, which is useful for practical purposes.

It is also possible to interpolate generalized Pareto processes with the same techniques as for an MSP, which is the topic of Section 4.1.

To begin with, in the following section we recall some basic theory needed in what follows and introduce some notation. For the ease of notation, we choose $K=[0,1]$ as the domain of the processes, being aware of the fact that all results are valid for an arbitrary compact set $K \subset \mathbb{R}$ as well. The extension of the results to more general domains (in particular, higher dimensions of the domain) is not immediately obvious and is the subject of current research; see Section 4.2 for an outline. 


\subsection{Prelimiaries}

We call an RV $\boldsymbol{X}=\left(X_{0}, \ldots, X_{d}\right)$ standard max-stable, if it is max-stable and each component follows the standard negative exponential distribution, i.e. $\mathbb{P}\left(X_{i} \leq x\right)=\exp (x)$, $x \leq 0, i=0, \ldots, d$. Different to that, a max-stable RV with unit Fréchet margins is commonly called simple max-stable in the literature; see, e.g. De Haan and Ferreira (2006). It is well known (see, e.g. De Haan and Resnick (1977), Pickands (1981), and Falk et al. (2011, Sections 4.2 and 4.3)) that $\boldsymbol{X}$ is standard max-stable if and only if (iff) there exists an $\mathrm{RV} \boldsymbol{Z}=\left(Z_{0}, \ldots, Z_{d}\right)$ with $Z_{i} \in[0, c]$ almost surely (a.s.) for some number $c \geq 1$, and $\mathbb{E}\left(Z_{i}\right)=1, i=0, \ldots, d$, such that

$$
\mathbb{P}(\boldsymbol{X} \leq \boldsymbol{x})=\exp \left(-\|\boldsymbol{x}\|_{D}\right):=\exp \left(-\mathbb{E}\left(\max _{0 \leq i \leq d}\left(\left|x_{i}\right| Z_{i}\right)\right)\right), \quad \boldsymbol{x} \leq \mathbf{0} \in \mathbb{R}^{d+1} .
$$

The condition $Z_{i} \in[0, c]$ a.s. can be weakened to $\mathbb{P}\left(Z_{i} \geq 0\right)=1$. Note that $\|\cdot\|_{D}$ defines a norm on $\mathbb{R}^{d+1}$, called the $D$-norm with generator $\boldsymbol{Z}$. The ' $D$ ' means dependence: we have independence of the margins of $\boldsymbol{X}$ iff $\|\cdot\|_{D}$ equals the norm $\|\boldsymbol{x}\|_{1}=\sum_{i=0}^{d}\left|x_{i}\right|$, which is generated by $\left(Z_{0}, \ldots, Z_{d}\right)$ being a random permutation of the vector $(d+1,0 \ldots, 0)$. We have complete dependence of the margins of $\boldsymbol{X}$ iff $\|\cdot\|_{D}$ is the maximum-norm $\|\boldsymbol{x}\|_{\infty}=$ $\max _{0 \leq i \leq d}\left|x_{i}\right|$, which is generated by the constant vector $\left(Z_{0} \ldots, Z_{d}\right)=(1, \ldots, 1)$. See Falk et al. (2011, Section 4.4) for further details of $D$-norms.

We call a stochastic process $\eta$ with sample paths in $\bar{C}^{-}[0,1]:=\{h \in C[0,1]: h \leq 0\}$ a standard max-stable process (SMSP) if it is an MSP with standard negative exponential univariate margins. Again, an MSP with unit Fréchet margins is commonly called a simple MSP in the literature; see, e.g. De Haan and Ferreira (2006).

Denote by $E[0,1]$ the set of those bounded functions $f:[0,1] \rightarrow \mathbb{R}$ that have only a finite number of discontinuities, and let $\bar{E}^{-}[0,1]$ be the subset of those functions in $E[0,1]$ which attain only nonpositive values.

From a mathematical point of view, it is quite convenient to introduce the space $E[0,1]$. It allows the incorporation of the finite-dimensional marginal distributions of a stochastic process $\boldsymbol{X}$ with sample paths in $\bar{C}^{-}[0,1]$ in the form $\mathbb{P}(\boldsymbol{X} \leq f)$ with a suitable choice of $f \in E[0,1]$. This entails the following characterization of an SMSP in terms of its distribution function, which is due to Giné et al. (1990): a stochastic process $\eta$ in $C[0,1]$ is an SMSP iff there exists a stochastic process $\boldsymbol{Z}=\left(Z_{t}\right)_{t \in[0,1]}$ with sample paths in $\bar{C}^{+}[0,1]:=\{g \in C[0,1]: g \geq 0\}$ with $Z_{t} \leq c$ a.s. for some constant $c \geq 1$, and $\mathbb{E}\left(Z_{t}\right)=1, t \in[0,1]$, such that

$$
\mathbb{P}(\eta \leq f)=\exp \left(-\|f\|_{D}\right):=\exp \left(-\mathbb{E}\left(\sup _{t \in[0,1]}\left(|f(t)| Z_{t}\right)\right)\right), \quad f \in \bar{E}^{-}[0,1] .
$$

A proper choice of the function $f \in \bar{E}^{-}[0,1]$ provides the finite-dimensional marginal distribution $\mathbb{P}(\eta \leq f)=\mathbb{P}\left(\eta_{t_{i}} \leq x_{i}, 1 \leq i \leq d\right)$.

The condition $\mathbb{P}\left(\sup _{t \in[0,1]} Z_{t} \leq c\right)=1$ on the generator process $\boldsymbol{Z}$ can be weakened to $E\left(\sup _{t \in[0,1]} Z_{t}\right)<\infty$; see De Haan and Ferreira (2006, Corollary 9.4.5).

Note that $\|\cdot\|_{D}$ defines a norm again, this time on the linear space $E[0,1]$. It is also called the $D$-norm with generator process $\boldsymbol{Z}$, and we have

$$
\|f\|_{\infty} \leq\|f\|_{D} \leq \varepsilon_{D}\|f\|_{\infty}, \quad f \in E[0,1],
$$

where $\|f\|_{\infty}=\sup _{t \in[0,1]}|f(t)|$ and $\varepsilon_{D}=\|1\|_{D}=\mathbb{E}\left(\|\boldsymbol{Z}\|_{\infty}\right)$ is the extremal coefficient, (cf. Smith (1990)). This implies that $\|\cdot\|_{D}=\|\cdot\|_{\infty}$ iff $\varepsilon_{D}=1$ (cf. Aulbach et al. (2013)). Moreover, the preceding inequality shows that each $D$-norm on the space $E[0,1]$ is equivalent to the sup norm $\|\cdot\|_{\infty}$, which is itself a $D$-norm by putting $Z_{t}=1, t \in[0,1]$. 


\section{The generalized max-linear model}

Let $\boldsymbol{X}=\left(X_{0}, \ldots, X_{d}\right)$ be a standard max-stable RV with pertaining $D$-norm $\|\cdot\|_{D_{0, \ldots, d}}$ on $\mathbb{R}^{d+1}$ generated by $\boldsymbol{Z}=\left(Z_{0}, \ldots, Z_{d}\right), d \in \mathbb{N}$, i.e.

$$
\mathbb{P}(\boldsymbol{X} \leq \boldsymbol{x})=\exp \left(-\|\boldsymbol{x}\|_{D_{0, \ldots, d}}\right)=\exp \left(-\mathbb{E}\left(\max _{i=0, \ldots, d}\left|x_{i}\right| Z_{i}\right)\right)
$$

$\boldsymbol{x}=\left(x_{0}, \ldots, x_{d}\right) \leq \mathbf{0}$. Choose arbitrary deterministic functions $g_{0}, \ldots, g_{d} \in \overline{\boldsymbol{C}}^{+}[0,1]$ with the property

$$
\left\|\left(g_{0}(t), \ldots, g_{d}(t)\right)\right\|_{D_{0, \ldots, d}}=1 \quad \text { for } t \in[0,1] .
$$

For instance, in the case of independent margins of $\boldsymbol{X}$, we have $\|\cdot\|_{D_{0, \ldots, d}}=\|\cdot\|_{1}$, and (1) becomes

$$
\sum_{i=0}^{d} g_{i}(t)=1 \text { for } t \in[0,1]
$$

i.e. $g_{i}(t), i=0, \ldots, d$ defines a probability distribution on the set $\{0, \ldots, d\}$ for each $t \in[0,1]$. This is the setup in the max-linear model introduced by Wang and Stoev (2011). An example for this case is given by the binomial distribution

$$
g_{i}(t):=\left(\begin{array}{c}
d \\
i
\end{array}\right) t^{i}(1-t)^{d-i} \quad \text { for } t \in[0,1], i=0, \ldots, d .
$$

Now

$$
\eta_{t}:=\max _{i=0, \ldots, d} \frac{X_{i}}{g_{i}(t)} \quad \text { for } t \in[0,1] .
$$

The model (2) is called the generalized max-linear model. It defines an SMSP, as we will show in the next lemma.

Lemma 1. The stochastic process $\eta=\left(\eta_{t}\right)_{t \in[0,1]}$ in (2) defines an SMSP with generator process $\hat{\mathbf{Z}}=\left(\hat{Z}_{t}\right)_{t \in[0,1]}$ given by

$$
\hat{Z}_{t}=\max _{i=0, \ldots, d}\left(g_{i}(t) Z_{i}\right) \quad \text { for } t \in[0,1] .
$$

Proof. First we verify that the process $\hat{\boldsymbol{Z}}$ is indeed a generator process. It is obvious that the sample paths of $\hat{\boldsymbol{Z}}$ are in $\overline{\boldsymbol{C}}^{+}[0,1]$. Furthermore, by construction for each $t \in[0,1]$, we have

$$
\mathbb{E}\left(\hat{Z}_{t}\right)=\left\|\left(g_{0}(t), \ldots, g_{d}(t)\right)\right\|_{D_{0, \ldots, d}}=1 .
$$

As $\|\cdot\|_{\infty} \leq\|\cdot\|_{D}$ for an arbitrary $D$-norm, we have $\left\|\left(g_{0}(t), \ldots, g_{d}(t)\right)\right\|_{\infty} \leq 1, t \in[0,1]$, and, thus, $\hat{Z}_{t} \leq \max _{i=0, \ldots, d} Z_{i}, t \in[0,1]$.

In addition, we have, for $f \in \bar{E}^{-}[0,1]$,

$$
\begin{aligned}
\mathbb{P}(\boldsymbol{\eta} \leq f) & =\mathbb{P}\left(X_{i} \leq g_{i}(t) f(t), i=0, \ldots, d, t \in[0,1]\right) \\
& =\exp \left(-\left\|\left(\sup _{t \in[0,1]}\left(g_{0}(t)|f(t)|\right), \ldots, \sup _{t \in[0,1]}\left(g_{d}(t)|f(t)|\right)\right)\right\|_{D_{0, \ldots, d}}\right) \\
& =\exp \left(-\mathbb{E}\left(\sup _{t \in[0,1]}\left(|f(t)| \max _{i=0, \ldots, d}\left(g_{i}(t) Z_{i}\right)\right)\right)\right) \\
& =\exp \left(-\mathbb{E}\left(\sup _{t \in[0,1]}\left(|f(t)| \hat{Z}_{t}\right)\right)\right),
\end{aligned}
$$

which completes the proof. 
Remark 1. Condition (1) ensures that the univariate margins $\eta_{t}, t \in[0,1]$, of the process $\eta$ in (2) follow the standard negative exponential distribution $\mathbb{P}\left(\eta_{t} \leq x\right)=\exp (x), x \leq 0$. If we drop this condition, we still obtain an MSP: take for $n \in \mathbb{N}$ independent and identically distributed copies $\boldsymbol{\eta}^{(1)}, \ldots, \boldsymbol{\eta}^{(n)}$ of $\boldsymbol{\eta}$. We have, for $f \in \bar{E}^{-}[0,1]$,

$$
\begin{aligned}
\mathbb{P}\left(n \max _{1 \leq k \leq n} \eta^{(k)} \leq f\right) & =\mathbb{P}\left(X_{i} \leq \inf _{t \in[0,1]}\left(\frac{g_{i}(t) f(t)}{n}\right), i=0, \ldots, d\right)^{n} \\
& =\exp \left(-\left\|\left(\sup _{t \in[0,1]}\left(g_{0}(t)|f(t)|\right), \ldots, \sup _{t \in[0,1]}\left(g_{d}(t)|f(t)|\right)\right)\right\|_{D_{0, \ldots, d}}\right) \\
& =\mathbb{P}(\boldsymbol{\eta} \leq f) .
\end{aligned}
$$

The univariate margins of $\boldsymbol{\eta}$ are now given by

$$
\mathbb{P}\left(\eta_{t} \leq x\right)=\exp \left(\left\|\left(g_{0}(t), \ldots, g_{d}(t)\right)\right\|_{D_{0, \ldots, d}} x\right) \quad \text { for } t \in[0,1], x \leq 0 .
$$

Note that the above calculations also provide an alternative proof of Lemma 1, except we do not obtain the generator process of $\boldsymbol{\eta}$ with this approach.

In (2) we have not made any further assumptions on the $D$-norm $\|\cdot\|_{D_{0, \ldots, d}}$, i.e. on the dependence structure of the RVs $X_{0}, \ldots, X_{d}$. The special case of $\|\cdot\|_{D_{0, \ldots, d}}=\|\cdot\|_{1}$ characterizes the independence of $X_{0}, \ldots, X_{d}$. This is the regular max-linear model (cf. Wang and Stoev (2011)).

On the contrary, $\|\cdot\|_{D_{0, \ldots, d}}=\|\cdot\|_{\infty}$ provides the case of complete dependence $X_{0}=\cdots=X_{d}$ a.s. with the constant generator $Z_{0}=\cdots=Z_{d}=1$. Thus, (1) becomes $\max _{i=0, \ldots, d} g_{i}(t)=1$, $t \in[0,1]$, and, therefore,

$$
\hat{Z}_{t}=\max _{i=0, \ldots, d}\left(g_{i}(t) Z_{i}\right)=\max _{i=0, \ldots, d} g_{i}(t)=1 \quad \text { for } t \in[0,1]
$$

\section{Reconstruction of an SMSP}

The preceding approach offers a way to reconstruct an SMSP in an appropriate way. Let $\boldsymbol{\eta}=\left(\eta_{t}\right)_{t \in[0,1]}$ be an SMSP with generator process $\boldsymbol{Z}=\left(Z_{t}\right)_{t \in[0,1]}$ and $D$-norm $\|\cdot\|_{D}$. Choose a grid $0=: s_{0}<s_{1}<\cdots<s_{d-1}<s_{d}:=1$ of points within $[0,1]$. Then $\left(\eta_{s_{0}}, \ldots, \eta_{s_{d}}\right)$ is a standard max-stable RV in $\mathbb{R}^{d+1}$ with pertaining $D$-norm $\|\cdot\|_{D_{0, \ldots, d}}$ generated by $\left(Z_{s_{0}}, \ldots, Z_{s_{d}}\right)$.

The aim in this section is to define some SMSP $\hat{\eta}=\left(\hat{\eta}_{t}\right)_{t \in[0,1]}$ for which $\hat{\eta}_{s_{i}}=\eta_{s_{i}}, i=$ $0, \ldots, d$, holds, i.e. $\hat{\eta}$ interpolates the finite-dimensional projections $\left(\eta_{s_{0}}, \ldots, \eta_{s_{d}}\right)$ of the original SMSP $\boldsymbol{\eta}$ in an appropriate way. This will be done by means of a special case of the generalized max-linear model, i.e. by a particular choice of the functions $g_{i}$ in (2). We show that this way of predicting the original MSP $\eta$ in space is reasonable, as the pointwise mean-squared error (MSE) $\left(\hat{\eta}_{t}^{(d)}\right):=\mathbb{E}\left(\left(\eta_{t}-\hat{\eta}_{t}^{(d)}\right)^{2}\right)$ diminishes for all $t \in[0,1]$ as $d$ increases. Moreover, we establish uniform convergence of the 'predictive' processes and the corresponding generator processes to the original one.

\subsection{Uniform convergence of the discretized versions}

As we have shown in Lemma 1, the stochastic process $\hat{\eta}=\left(\hat{\eta}_{t}\right)_{t \in[0,1]}$,

$$
\hat{\eta}_{t}=\max _{i=0, \ldots, d} \frac{\eta_{s_{i}}}{g_{i}(t)} \quad \text { for } t \in[0,1]
$$


defines an SMSP with generator process $\hat{Z}=\left(\hat{Z}_{t}\right)_{t \in[0,1]}$, given by

$$
\hat{Z}_{t}=\max _{i=0, \ldots, d}\left(g_{i}(t) Z_{s_{i}}\right) \quad \text { for } t \in[0,1]
$$

for arbitrary functions $g_{0}, \ldots, g_{d} \in \bar{C}^{+}[0,1]$ that satisfy (1). We are going to specialize them now.

Denote by $\|\cdot\|_{D_{i-1, i}}$ the $D$-norm pertaining to the bivariate RV $\left(\eta_{s_{i-1}}, \eta_{s_{i}}\right), i=1, \ldots, d$. Put, $i=1, \ldots, d-1$,

$$
\begin{aligned}
& g_{0}^{*}(t):= \begin{cases}\frac{s_{1}-t}{\left\|\left(s_{1}-t, t\right)\right\|_{D_{0,1}}} & \text { for } t \in\left[0, s_{1}\right], \\
0 & \text { otherwise, }\end{cases} \\
& g_{i}^{*}(t):= \begin{cases}\frac{t-s_{i-1}}{\left\|\left(s_{i}-t, t-s_{i-1}\right)\right\|_{D_{i-1, i}}} & \text { for } t \in\left[s_{i-1}, s_{i}\right], \\
\frac{s_{i+1}-t}{\left\|\left(s_{i+1}-t, t-s_{i}\right)\right\|_{D_{i, i+1}}} & \text { for } t \in\left[s_{i}, s_{i+1}\right], \\
0 & \text { otherwise, }\end{cases} \\
& g_{d}^{*}(t):= \begin{cases}\frac{t-s_{d-1}}{\left\|\left(s_{d}-t, t-s_{d-1}\right)\right\|_{D_{d-1, d}}} & \text { for } t \in\left[s_{d-1}, s_{d}\right], \\
0 & \text { otherwise. }\end{cases}
\end{aligned}
$$

Clearly, $g_{0}^{*}, \ldots, g_{d}^{*} \in \bar{C}^{+}[0,1]$ since the fact that a $D$-norm is standardized implies that

$$
\lim _{t \uparrow s_{i}} g_{i}^{*}(t)=\frac{s_{i}-s_{i-1}}{\left\|\left(0, s_{i}-s_{i-1}\right)\right\|_{D_{i-1, i}}}=1=\frac{s_{i+1}-s_{i}}{\left\|\left(s_{i+1}-s_{i}, 0\right)\right\|_{D_{i-1, i}}}=\lim _{t \downarrow s_{i}} g_{i}^{*}(t) .
$$

Moreover, we have

$$
\left\|\left(g_{0}^{*}(t), \ldots, g_{d}^{*}(t)\right)\right\|_{D_{0, \ldots, d}}=\left\|\left(g_{i-1}^{*}(t), g_{i}^{*}(t)\right)\right\|_{D_{i-1, i}}=1 \quad \text { for } t \in\left[s_{i-1}, s_{i}\right], i=1, \ldots, d .
$$

Hence, the functions $g_{0}^{*}, \ldots, g_{d}^{*}$ are suitable for the generalized max-linear model (2). In addition, they have the following property.

Lemma 2. The functions $g_{0}^{*}, \ldots, g_{d}^{*}$ defined above satisfy

$$
\left\|g_{i}^{*}\right\|_{\infty}=g_{i}^{*}\left(s_{i}\right)=1, \quad i=0, \ldots, d .
$$

In view of their properties described above, the functions $g_{i}^{*}$ can be viewed as kernel functions quite similar to kernels in nonparametric kernel density estimators. Each function $g_{i}^{*}(t)$ has maximum value 1 at $t=s_{i}$ and, with the distance between $t$ and $s_{i}$ increasing, the value $g_{i}^{*}(t)$ shrinks to 0 . This view also provides the idea behind the extension of this approach to higher dimensions as outlined in Section 4.2.

Proof. From the fact that a $D$-norm is monotone and standardized, we obtain for $i=$ $1, \ldots, d-1$ and $t \in\left[s_{i-1}, s_{i}\right)$,

$$
g_{i}^{*}(t)=\frac{t-s_{i-1}}{\left\|\left(s_{i}-t, t-s_{i-1}\right)\right\|_{D_{i-1, i}}}=\left[\left\|\left(\frac{s_{i}-t}{t-s_{i-1}}, 1\right)\right\|_{D_{i-1, i}}\right]^{-1} \leq \frac{1}{\|(0,1)\|_{D_{i-1, i}}}=1,
$$


and, for $t \in\left[s_{i}, s_{i+1}\right)$,

$$
g_{i}^{*}(t)=\frac{s_{i+1}-t}{\left\|\left(s_{i+1}-t, t-s_{i}\right)\right\|_{D_{i, i+1}}}=\left[\left\|\left(1, \frac{t-s_{i}}{s_{i+1}-t}\right)\right\|_{D_{i, i+1}}\right]^{-1} \leq \frac{1}{\|(1,0)\|_{D_{i, i+1}}}=1 .
$$

Analogously, we have $g_{0}^{*} \leq 1$ and $g_{d}^{*} \leq 1$. The assertion now follows since $g_{i}^{*}\left(s_{i}\right)=1$, $i=0, \ldots, d$.

The SMSP $\hat{\eta}=\left(\hat{\eta}_{t}\right)_{t \in[0,1]}$ that is generated by the generalized max-linear model with these particular functions $g_{0}^{*}, \ldots, g_{d}^{*}$ is given by

$$
\begin{aligned}
\hat{\eta}_{t} & =\max \left(\frac{\eta_{s_{i-1}}}{g_{i-1}^{*}(t)}, \frac{\eta_{s_{i}}}{g_{i}^{*}(t)}\right) \\
& =\left\|\left(s_{i}-t, t-s_{i-1}\right)\right\|_{D_{i-1, i}} \max \left(\frac{\eta_{s_{i-1}}}{s_{i}-t}, \frac{\eta_{s_{i}}}{t-s_{i-1}}\right) \text { for } t \in\left[s_{i-1}, s_{i}\right], i=1, \ldots, d .
\end{aligned}
$$

Note that $\eta_{s_{i}}<0$ a.s., $i=0, \ldots, d$. This implies that the maximum taken over $d+1$ points in (2) goes down to a maximum taken over only two points in (3) since all except two of the $g_{i}$ vanish in $t \in\left[s_{i-1}, s_{i}\right], i=1, \ldots, d$. We have, moreover,

$$
\hat{\eta}_{s_{i}}=\eta_{s_{i}}, \quad i=0, \ldots, d,
$$

so the above process interpolates the RV $\left(\eta_{s_{0}}, \ldots, \eta_{s_{d}}\right)$.

To give an example, put $\left\|\left(x_{1}, x_{2}\right)\right\|_{D_{i-1, i}}:=\left\|\left(x_{1}, x_{2}\right)\right\|_{\lambda}:=\left(\left|x_{1}\right|^{\lambda}+\left|x_{2}\right|^{\lambda}\right)^{1 / \lambda}, 1 \leq \lambda \leq \infty$ for every $1 \leq i \leq d$, i.e. each bivariate $D$-norm $\|\cdot\|_{D_{i-1, i}}$ is the logistic one. For $1<\lambda<\infty$, the logistic $D$-norm is generated by $Z_{i}:=X_{i} / \Gamma\left(1-\lambda^{-1}\right), i=1,2$, where $X_{1}$ and $X_{2}$ are independent and identically unit Fréchet distributed $\mathrm{RVs}$ and $\Gamma(\cdot)$ denotes the gamma function. In this case, we obtain the representation

$$
\hat{\eta}_{t}=\left(\left(s_{i}-t\right)^{\lambda}+\left(t-s_{i-1}\right)^{\lambda}\right)^{1 / \lambda} \max \left(\frac{\eta_{s_{i-1}}}{s_{i}-t}, \frac{\eta_{s_{i}}}{t-s_{i-1}}\right) \text { for } t \in\left[s_{i-1}, s_{i}\right], i=1, \ldots, d .
$$

In summary, we have proved the following result.

Corollary 1. Let $\boldsymbol{\eta}=\left(\eta_{t}\right)_{t \in[0,1]}$ be an SMSP with generator $\boldsymbol{Z}=\left(Z_{t}\right)_{t \in[0,1]}$, and let $0:=$ $s_{0}<s_{1}<, \ldots,<s_{d-1}<s_{d}:=1$ be a grid of points in the interval [0,1]. The process $\hat{\boldsymbol{\eta}}=\left(\hat{\eta}_{t}\right)_{t \in[0,1]}$ defined in (3) is an SMSP with generator process $\hat{\boldsymbol{Z}}=\left(\hat{Z}_{t}\right)_{t \in[0,1]}$, where

$$
\hat{Z}_{t}=\frac{\max \left(\left(s_{i}-t\right) Z_{s_{i-1}},\left(t-s_{i-1}\right) Z_{s_{i}}\right)}{\left\|\left(s_{i}-t, t-s_{i-1}\right)\right\|_{D_{i-1, i}}} \text { for } t \in\left[s_{i-1}, s_{i}\right], i=1, \ldots, d .
$$

The processes $\hat{\boldsymbol{\eta}}$ and $\hat{\boldsymbol{Z}}$ interpolate the $R V\left(\eta_{s_{0}}, \ldots, \eta_{s_{d}}\right)$ and $\left(Z_{s_{0}}, \ldots, Z_{s_{d}}\right)$, respectively.

We call $\hat{\boldsymbol{\eta}}$ the discretized version of $\boldsymbol{\eta}$ and $\hat{\boldsymbol{Z}}$ the discretized version of $\boldsymbol{Z}$, both with grid $\left\{s_{0}, \ldots, s_{d}\right\}$. Next, we show that the preceding approach allows the approximation of an underlying SMSP based on multivariate observations; i.e. the discretized version of the underlying SMSP converges to this very process in a strong sense. We need the following two lemmas which provide some technical insight into the structure of the chosen max-linear model. 
Lemma 3. The SMSP defined in (3) fulfills

$$
\sup _{t \in\left[s_{i-1}, s_{i}\right]} \hat{\eta}_{t}=\max \left(\eta_{s_{i-1}}, \eta_{s_{i}}\right), \quad \inf _{t \in\left[s_{i-1}, s_{i}\right]} \hat{\eta}_{t}=-\left\|\left(\eta_{s_{i-1}}, \eta_{s_{i}}\right)\right\|_{D_{s_{i-1}, s_{i}}} \text { for } i=1, \ldots, d .
$$

This minimum is attained for $t=\left(s_{i-1} \eta_{s_{i-1}}+s_{i} \eta_{s_{i}}\right) /\left(\eta_{s_{i-1}}+\eta_{s_{i}}\right)$.

Proof. We know from Lemma 2 that $g_{i-1}^{*}(t), g_{i}^{*}(t) \leq 1$ for an arbitrary $i=1, \ldots, d$ and $t \in\left[s_{i-1}, s_{i}\right]$. Hence,

$$
\hat{\eta}_{t}=\max \left(\frac{\eta_{s_{i-1}}}{g_{i-1}^{*}(t)}, \frac{\eta_{s_{i}}}{g_{i}^{*}(t)}\right) \leq \max \left(\eta_{s_{i-1}}, \eta_{s_{i}}\right) \quad \text { for } t \in\left[s_{i-1}, s_{i}\right], i=1, \ldots, d,
$$

which yields the first part of the assertion. Recall that $\eta_{s_{i}}<0$ with probability $1, i=0 \ldots, d$.

Moreover, we have, for $t \in\left(s_{i-1}, s_{i}\right)$,

$$
\frac{\eta_{s_{i-1}}}{s_{i}-t} \leq \frac{\eta_{s_{i}}}{t-s_{i-1}} \quad \Longleftrightarrow \quad \frac{s_{i}-t}{t-s_{i-1}} \leq \frac{\eta_{s_{i-1}}}{\eta_{s_{i}}} \Longleftrightarrow t \geq \frac{s_{i-1} \eta_{s_{i-1}}+s_{i} \eta_{s_{i}}}{\eta_{s_{i-1}}+\eta_{s_{i}}},
$$

where equality in one of these expressions occurs iff it does in the other two. In this case of equality, we have

$$
\hat{\eta}_{t}=\left\|\left(s_{i}-t, t-s_{i-1}\right)\right\|_{D_{i-1, i}} \frac{\eta_{s_{i}}}{t-s_{i-1}}=-\left\|\left(\eta_{s_{i-1}}, \eta_{s_{i}}\right)\right\|_{D_{i-1, i}} .
$$

On the other hand, the monotonicity of a $D$-norm implies for every $t \in\left(s_{i-1}, s_{i}\right)$ with $t \geq$ $\left(s_{i-1} \eta_{s_{i-1}}+s_{i} \eta_{s_{i}}\right) /\left(\eta_{s_{i-1}}+\eta_{s_{i}}\right)$,

$$
\begin{aligned}
\hat{\eta}_{t} & \geq\left\|\left(s_{i}-t, t-s_{i-1}\right)\right\|_{D_{i-1, i}} \frac{\eta_{s_{i}}}{t-s_{i-1}} \\
& =\left\|\left(\frac{s_{i}-t}{t-s_{i-1}}, 1\right)\right\|_{D_{i-1, i}} \eta_{s_{i}} \\
& \geq\left\|\left(\frac{\eta_{s_{i-1}}}{\eta_{s_{i}}}, 1\right)\right\|_{D_{i-1, i}} \eta_{s_{i}} \\
& =-\left\|\left(\eta_{s_{i-1}}, \eta_{s_{i}}\right)\right\|_{D_{i-1, i}} .
\end{aligned}
$$

Recall again that $\eta_{s_{i}}<0$ a.s. The $t \leq\left(s_{i-1} \eta_{s_{i-1}}+s_{i} \eta_{s_{i}}\right) /\left(\eta_{s_{i-1}}+\eta_{s_{i}}\right)$ case works analogously.

As an immediate consequence of the preceding result, we obtain

$$
\hat{\eta} \leq x \quad \Longleftrightarrow \quad \max \left(\eta_{s_{0}}, \ldots, \eta_{s_{d}}\right) \leq x
$$

and

$$
\hat{\boldsymbol{\eta}}>x \Longleftrightarrow \max _{1 \leq i \leq d}\left\|\left(\eta_{s_{i-1}}, \eta_{s_{i}}\right)\right\|_{D_{i-1, i}}<-x \quad \text { for } x \leq 0 .
$$

In order to visualize the interpolation scheme of this particular generalized max-linear model, we plot some discretized versions with different grids and bivariate $D$-norms $\|\cdot\|_{D_{i-1, i}}$. For the sake of simplicity, the underlying path $\eta(\omega)$ in this example does not arise from a simulation of an actual SMSP, but rather is replaced by a smooth deterministic continuous function on $[0,1]$. 

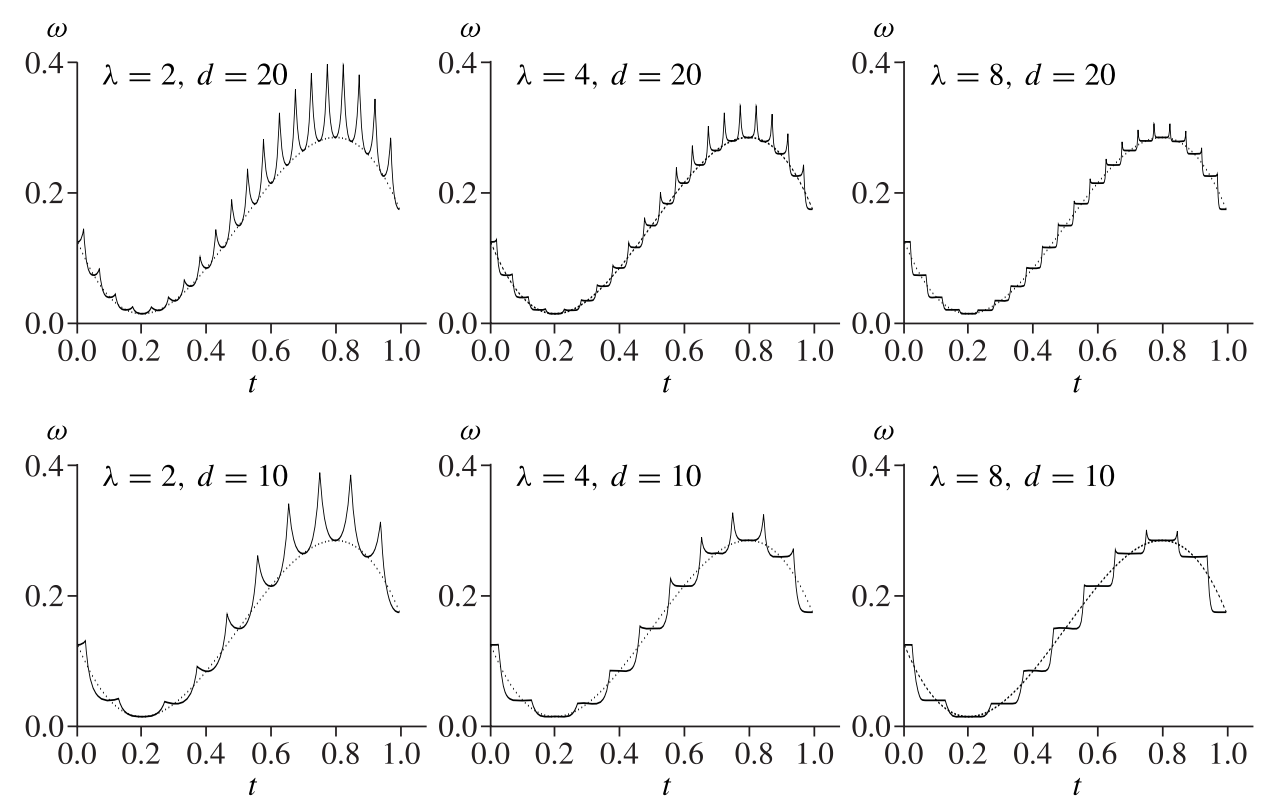

$\omega$

$\omega$
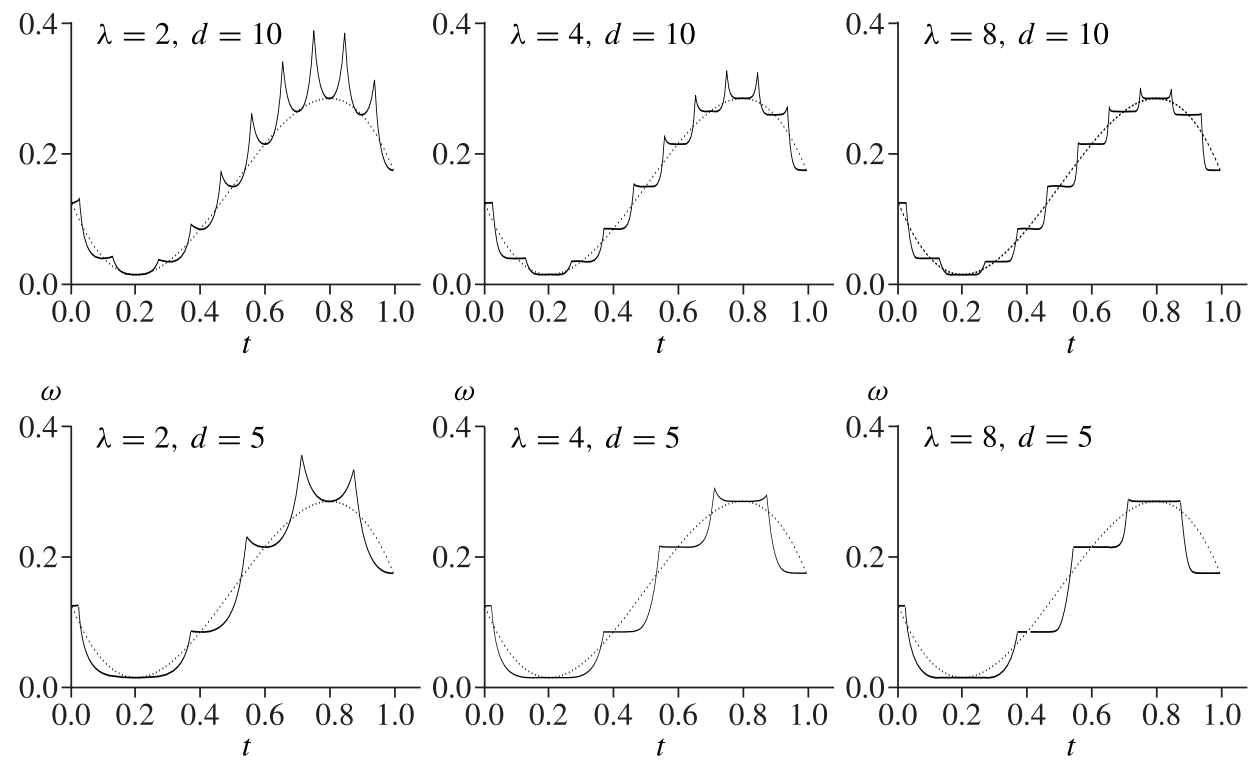

FIGURE 1: Plots of logistic type discretized versions (solid) of a deterministic function that stands for a path of an SMSP (dotted).

More precisely, in Figure 1, we choose $\eta_{t}(\omega):=7.5\left(0.16 t-0.5 t^{2}+t^{3} / 3\right)-0.125, t \in[0,1]$, which is represented by the dotted curve. The solid line in each plot is the discretized version $\hat{\eta}(\omega)$ of this path. We use equidistant grids of dimension $d=5, d=10$, and $d=20$. Each bivariate $D$-norm $\|\cdot\|_{D_{i-1, i}}$ are logistic norms such that the discretized versions are given by (4) with $\lambda=2, \lambda=4$, and $\lambda=8$.

The plots apparently show that the approximation of the original process through a discretized version improves as the dimension $d$ increases and as the bivariate $D$-norms get closer to the complete dependence case.

The next lemma concerns the structure of the underlying generator processes. It can easily be proved by arguments that are very similar to those of the proof of Lemma 2.

Lemma 4. The generator process defined in (5) fulfills for $i=1, \ldots, d$,

$$
\sup _{t \in\left[s_{i-1}, s_{i}\right]} \hat{Z}_{t}=\max \left(Z_{s_{i-1}}, Z_{s_{i}}\right) \text {. }
$$


In particular, the extremal coefficient $\mathbb{E}\left(\|\hat{\mathbf{Z}}\|_{\infty}\right)$ of the SMSP $\hat{\boldsymbol{\eta}}$ coincides with the extremal coefficient $\mathbb{E}\left(\max _{i=0, \ldots, d} Z_{s_{i}}\right)$ of the $R V\left(\eta_{s_{0}}, \ldots, \eta_{s_{d}}\right)$. Moreover, for $i=1, \ldots, d$,

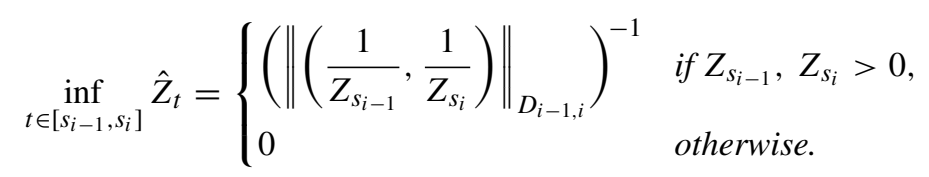

In the first case, the minimum is attained for $t=\left(s_{i-1} Z_{s_{i}}+s_{i} Z_{s_{i-1}}\right) /\left(Z_{s_{i-1}}+Z_{s_{i}}\right)$.

So far we have only considered a fixed discretized version of an SMSP. The next step is to examine a sequence of discretized versions with certain grids whose diameter converges to 0 . It turns out that such a sequence converges to the initial SMSP in the function space $C[0,1]$ equipped with the sup norm. Thus, our method is suitable to reconstruct the initial process.

Let

$$
g_{d}:=\left\{s_{0}^{(d)}, s_{1}^{(d)} \ldots, s_{d}^{(d)}\right\}, \quad 0=: s_{0}^{(d)}<s_{1}^{(d)}<\cdots<s_{d}^{(d)}:=1, \quad d \in \mathbb{N},
$$

be a sequence of grids in $[0,1]$ with diameter

$$
\kappa_{d}:=\max _{i=1, \ldots, d}\left(s_{i}^{(d)}-s_{i-1}^{(d)}\right) \longrightarrow 0 \quad \text { as } d \rightarrow \infty .
$$

Let $\hat{\boldsymbol{\eta}}^{(d)}=\left(\hat{\eta}_{t}^{(d)}\right)_{t \in[0,1]}$ be the discretized version of an SMSP $\boldsymbol{\eta}=\left(\eta_{t}\right)_{t \in[0,1]}$ with grid $g_{d}$. Denote by $\hat{\boldsymbol{Z}}^{(d)}=\left(\hat{Z}_{t}^{(d)}\right)_{t \in[0,1]}$ and $\boldsymbol{Z}=\left(Z_{t}\right)_{t \in[0,1]}$ the generator processes pertaining to $\hat{\boldsymbol{\eta}}^{(d)}$ and $\eta$, respectively.

Theorem 1. The processes $\hat{\boldsymbol{\eta}}^{(d)}$ and $\hat{\boldsymbol{Z}}^{(d)}, d \in \mathbb{N}$, converge uniformly to $\boldsymbol{\eta}$ and $\boldsymbol{Z}$ pathwise, i.e. $\left\|\hat{\boldsymbol{\eta}}^{(d)}-\boldsymbol{\eta}\right\|_{\infty} \rightarrow 0$ and $\left\|\hat{\boldsymbol{Z}}^{(d)}-\boldsymbol{Z}\right\|_{\infty} \rightarrow 0$ as $d \rightarrow \infty$ with probability 1 .

Proof. Denote by $[t]_{d}, d \in \mathbb{N}$, the left neighbor of $t \in[0,1]$ among $g_{d}$, and by $\langle t\rangle_{d}, d \in \mathbb{N}$, the right neighbor of $t \in[0,1]$ among $g_{d}$. Choose a sequence $s^{(d)} \in[0,1], d \in \mathbb{N}$ with $s^{(d)} \rightarrow s \in[0,1], d \in N$. Then obviously $\left[s^{(d)}\right]_{d} \rightarrow s$ and $\left\langle s^{(d)}\right\rangle_{d} \rightarrow s$ as $d \rightarrow \infty$. Hence, by Lemma 3 and the continuity of the process $\eta$, we obtain

$$
\hat{\eta}_{s^{(d)}}^{(d)} \leq \max _{s \in\left[\left[s^{(d)}\right]_{d},\left\langle s^{(d)}\right\rangle_{d}\right]} \hat{\eta}_{s}^{(d)}=\max \left(\eta_{\left[s^{(d)}\right]_{d}}, \eta_{\left\langle s^{(d)}\right\rangle_{d}}\right) \longrightarrow \eta_{s} \quad \text { as } d \rightarrow \infty .
$$

as well as

$$
\hat{\eta}_{s^{(d)}}^{(d)} \geq \min _{s \in\left[\left[s^{(d)}\right]_{d},\langle s(d)\rangle_{d}\right]} \hat{\eta}_{s}^{(d)}=-\left\|\left(\eta_{\left[s^{(d)}\right]_{d}}, \eta_{\left\langle s^{(d)}\right\rangle_{d}}\right)\right\|_{\left.D_{[s(d)]_{d},\langle s}(d)\right\rangle_{d}} \longrightarrow \eta_{s} \quad \text { as } d \rightarrow \infty,
$$

where $\|\cdot\|_{\left.D_{[s}(d)\right]_{d}\langle s(d)\rangle_{d}}$ denotes the $D$-norm pertaining to $\left(\eta_{\left[s^{(d)}\right]_{d}}, \eta_{\left\langle s^{(d)}\right\rangle_{d}}\right)$. Hence, the first part of the assertion is proven.

Now we show that $\hat{\boldsymbol{Z}}^{(d)} \rightarrow \boldsymbol{Z} \in\left(C[0,1],\|\cdot\|_{\infty}\right)$ as $d \rightarrow \infty$. If $Z_{s} \neq 0$, the continuity of $\boldsymbol{Z}$ implies that $Z_{\left[s^{(d)}\right]_{d}} \neq 0 \neq Z_{\left\langle s^{(d)}\right\rangle_{d}}$ for sufficiently large values of $d$. Repeating the above arguments, the assertion now follows by Lemma 4 . If $Z_{s}=0$, the continuity of $\boldsymbol{Z}$ implies that

$$
\hat{Z}_{s^{(d)}}^{(d)} \leq 2 \max \left(Z_{\left[s^{(d)}\right]_{d}}, Z_{\left\langle s^{(d)}\right\rangle_{d}}\right) \longrightarrow 2 Z_{s}=0 \quad \text { as } d \rightarrow \infty,
$$

which completes the proof. Check that $\left\|\left(\left\langle s^{(d)}\right\rangle_{d}-t, t-\left[s^{(d)}\right]_{d}\right)\right\|_{D} \geq \frac{1}{2}$ since every $D$-norm is monotone and standardized. 
The preceding theorem is the main reason why we consider the discretized version $\hat{\eta}$ of an SMSP $\eta$ a reasonable predictor of this process where the prediction is done in space, not in time. The predictions $\hat{\eta}_{t}$ of the points $\eta_{t}, t \in[0,1]$, depend only on the multivariate observations $\left(\eta_{s_{0}}, \ldots, \eta_{s_{d}}\right)$. More precisely, the only additional thing we need to know to make these predictions is the set of the adjacent bivariate marginal distributions of $\left(\eta_{s_{0}}, \ldots, \eta_{s_{d}}\right)$, i.e. the bivariate $D$-norms $\|\cdot\|_{D_{i-1, i}}, i=1, \ldots, d$. This might, however, be a restrictive condition in praxis and suggests the problem of fitting models of bivariate $D$-norms to data, which is, however, beyond the scope of this paper and requires future investigation.

The following results, however, are obvious. Let $\hat{\eta}_{t}$ be a point of the discretized version defined in (3) and define a defective discretized version via

$$
\tilde{\eta}_{t}:=\left\|\left(s_{i}-t, t-s_{i-1}\right)\right\|_{\tilde{D}_{i}} \max \left(\frac{\eta_{s_{i-1}}}{s_{i}-t}, \frac{\eta_{s_{i}}}{t-s_{i-1}}\right), \quad t \in\left[s_{i-1}, s_{i}\right], i=1, \ldots, d,
$$

where $\|\cdot\|_{\tilde{D}_{i}}$ is an arbitrary $D$-norm on $\mathbb{R}^{2}$ which we call the defective norm. Then for every $t \in\left[s_{i-1}, s_{i}\right], i=1, \ldots, d$,

$$
\left|\hat{\eta}_{t}-\tilde{\eta}_{t}\right|=\left|\left\|\left(s_{i}-t, t-s_{i-1}\right)\right\|_{D_{i-1, i}}-\left\|\left(s_{i}-t, t-s_{i-1}\right)\right\|_{\tilde{D}_{i}}\right| \min \left(\frac{-\eta_{s_{i-1}}}{s_{i}-t}, \frac{-\eta_{s_{i}}}{t-s_{i-1}}\right) .
$$

In particular, we have $\tilde{\eta}_{s_{i}}=\hat{\eta}_{s_{i}}=\eta_{s_{i}}, i=0, \ldots, d$. This means that we obtain an interpolating process even if we replace the $D$-norm $\|\cdot\|_{D_{i-1, i}}$ by the defective norm $\|\cdot\|_{\tilde{D}_{i}}$. Furthermore, the defective discretized version still defines an MSP with sample paths in $\bar{C}^{-}[0,1]$. Its univariate marginal distributions are given by

$$
\mathbb{P}\left(\tilde{\eta}_{t} \leq x\right)=\exp \left(\frac{\left\|\left(s_{i}-t, t-s_{i-1}\right)\right\|_{D_{i-1, i}}}{\left\|\left(s_{i}-t, t-s_{i-1}\right)\right\|_{\tilde{D}_{i}}} x\right), \quad x \leq 0, t \in\left[s_{i-1}, s_{i}\right], i=1, \ldots, d .
$$

In addition to this, the assertions in Lemma 3 also hold for the defective discretized version as each defective norm $\|\cdot\|_{\tilde{D}_{i}}$ is monotone and standardized. Repeating the arguments in the proof of Theorem 1 now shows that the uniform convergence towards the original process $\eta$ is retained if we replace the norms $\|\cdot\|_{D_{i-1, i}}$ by arbitrary monotone and standardized norms $\|\cdot\|_{\tilde{D}_{i}}$. In this case, the only property of the discretized version that we have to drop is the standardization of the univariate margins.

\subsection{The mean-squared error of the discretized version}

Considering $\hat{\eta}_{t}$ a predictor of $\eta_{t}$, we can ask for further properties such as the mean-squared error, which is our next aim. For that purpose, we formulate the following lemma. It applies to bivariate standard max-stable RVs in general. For a proof of this lemma; see Falk et al. (2014).

Lemma 5. Let $(X, Y)$ be a bivariate standard max-stable RV, i.e. there exists some D-norm $\|\cdot\|_{D}$ such that $\mathbb{P}(X \leq x, Y \leq y)=\exp \left(-\|(x, y)\|_{D}\right), x, y \leq 0$. Then

$$
E(X Y)=\int_{0}^{\infty} \frac{1}{\|(1, t)\|_{D}^{2}} \mathrm{~d} t
$$

In particular, the covariance and the correlation coefficient $\varrho$ of $X$ and $Y$ are given by

$$
\operatorname{cov}(X, Y)=\int_{0}^{\infty} \frac{1}{\|(1, t)\|_{D}^{2}} \mathrm{~d} t-1=\varrho(X, Y)
$$


Example 1. In accordance with the characterization of the independence and complete dependence case in terms of $D$-norms, in the case $\|\cdot\|_{D}=\|\cdot\|_{1}$, we obtain

$$
\operatorname{cov}(X, Y)=\int_{0}^{\infty} \frac{1}{(u+1)^{2}} \mathrm{~d} u-1=0
$$

and in case of $\|\cdot\|_{D}=\|\cdot\|_{\infty}$, we have

$$
\operatorname{cov}(X, Y)=\int_{0}^{\infty} \frac{1}{(\max (u, 1))^{2}} \mathrm{~d} u-1=1
$$

In particular, we have $\operatorname{cov}(X, Y)=\varrho(X, Y) \in[0,1]$ for every bivariate standard max-stable $\mathrm{RV}(X, Y)$ since the maximum norm is the least $D$-norm and the sum norm is the largest $D$-norm. In addition to this, for a general logistic $D$-norm $\|\cdot\|_{\lambda}$ with parameter $\lambda \in[1, \infty)$, by substituting $u \mapsto u^{1 / \lambda}$, we obtain

$$
\operatorname{cov}(X, Y)=\int_{0}^{\infty} \frac{1}{\left(u^{\lambda}+1\right)^{2 / \lambda}} \mathrm{d} u-1=\frac{1}{\lambda} \int_{0}^{\infty} \frac{u^{1 / \lambda-1}}{(u+1)^{2 / \lambda}} \mathrm{d} u-1=\frac{1}{\lambda} B\left(\frac{1}{\lambda}, \frac{1}{\lambda}\right)-1,
$$

where $B(x, y)=\int_{0}^{1} u^{x-1}(1-u)^{y-1} \mathrm{~d} u=\int_{0}^{\infty}\left(u^{x-1} /(1+u)^{x+y}\right) \mathrm{d} u$ denotes the Euler beta function.

In order to calculate the mean-squared error of the predictor $\hat{\eta}_{t}$, we have to determine the mixed moment $\mathbb{E}\left(\eta_{t} \hat{\eta}_{t}\right)$. For this purpose, we want to apply the previous lemma, which is why we need to ensure that the vector $\left(\eta_{t}, \hat{\eta}_{t}\right)$ is standard max-stable itself. This is the content of the following lemma.

Lemma 6. Let $\boldsymbol{\eta}=\left(\eta_{t}\right)_{t \in[0,1]}$ be an SMSP and denote by $\hat{\boldsymbol{\eta}}=\left(\hat{\eta}_{t}\right)_{t \in[0,1]}$ its discretized version with grid $\left\{s_{0}, \ldots, s_{d}\right\}$. Then the bivariate $R V\left(\eta_{t}, \hat{\eta}_{t}\right)$ is standard max-stable for every $t \in[0,1]$ with D-norm of the two-dimensional marginal

$$
\|(x, y)\|_{D_{t}}:=\left\|\left(x, g_{i-1}^{*}(t) y, g_{i}^{*}(t) y\right)\right\|_{D_{t, i-1, i}}, \quad t \in\left[s_{i-1}, s_{i}\right], i=1, \ldots, d,
$$

where $\|\cdot\|_{D_{t, i-1, i}}$ is the D-norm pertaining to $\left(\eta_{t}, \eta_{s_{i-1}}, \eta_{s_{i}}\right)$.

Proof. We have for every $t \in\left[s_{i-1}, s_{i}\right], x, y \leq 0$ and $i=1, \ldots, d$,

$$
\begin{aligned}
\mathbb{P}\left(\eta_{t} \leq x, \hat{\eta}_{t} \leq y\right) & =\mathbb{P}\left(\eta_{t} \leq x, \eta_{s_{i-1}} \leq g_{i-1}^{*}(t) y, \eta_{s_{i}} \leq g_{i}^{*}(t) y\right) \\
& =\exp \left(-\mathbb{E}\left(\max \left(|x| Z_{t}, g_{i-1}^{*}(t)|y| Z_{s_{i-1}}, g_{i}^{*}(t)|y| Z_{s_{i}}\right)\right)\right) \\
& =\exp \left(-\mathbb{E}\left(\max \left(|x| Z_{t},|y| \max \left(g_{i-1}^{*}(t) Z_{s_{i-1}}, g_{i}^{*}(t) Z_{s_{i}}\right)\right)\right)\right) .
\end{aligned}
$$

The vector

$$
\left(Z_{t}, \max \left(g_{i-1}^{*}(t) Z_{s_{i-1}}, g_{i}^{*}(t) Z_{s_{i}}\right)\right)
$$

defines a generator for every $t \in\left[s_{i-1}, s_{i}\right], i=1, \ldots, d$ as, for all such $t$,

$$
\mathbb{E}\left(\max \left(g_{i-1}^{*}(t) Z_{s_{i-1}}, g_{i}^{*}(t) Z_{s_{i}}\right)\right)=\left\|\left(g_{i-1}^{*}(t), g_{i}^{*}(t)\right)\right\|_{D_{i-1, i}}=1 .
$$

Let us recall the sequence of processes we have discussed in Theorem 1. Suppose $\eta$ is an SMSP and choose a sequence of grids $g_{d}$ of the interval $[0,1]$ with diameter $\kappa_{d} \rightarrow 0$ as $d \rightarrow \infty$. Denote by $\hat{\boldsymbol{\eta}}^{(d)}, d \in \mathbb{N}$, the sequence of dicretized versions of $\boldsymbol{\eta}$ with grid $g_{d}$. Denote further by $\|\cdot\|_{D_{t}^{(d)}}$ the $D$-norm pertaining to $\left(\eta_{t}, \hat{\eta}_{t}^{(d)}\right), t \in[0,1]$, and $d \in \mathbb{N}$. 
Theorem 2. Let $\boldsymbol{\eta}$ and $\hat{\boldsymbol{\eta}}^{(d)}, d \in \mathbb{N}$, be as above. The MSE of $\hat{\eta}_{t}^{(d)}$ is given by

$$
\operatorname{MSE}\left(\hat{\eta}_{t}^{(d)}\right):=\mathbb{E}\left(\left(\eta_{t}-\hat{\eta}_{t}^{(d)}\right)^{2}\right)=2\left(2-\int_{0}^{\infty} \frac{1}{\|(1, u)\|_{D_{t}^{(d)}}^{2}} \mathrm{~d} u\right) \longrightarrow 0 \quad \text { as } d \rightarrow \infty
$$

Proof. The second moment of a standard negative exponentially distributed RV is 2 and, therefore, by Lemma 5 and Lemma 6, we obtain

$$
\operatorname{MSE}\left(\eta_{t}^{(d)}\right)=\mathbb{E}\left(\eta_{t}^{2}\right)-2 \mathbb{E}\left(\eta_{t} \hat{\eta}_{t}^{(d)}\right)+\mathbb{E}\left(\left(\hat{\eta}_{t}^{(d)}\right)^{2}\right)=4-2 \int_{0}^{\infty} \frac{1}{\|(1, u)\|_{D_{t}^{(d)}}^{2}} \mathrm{~d} u .
$$

Next, we show that $\|\cdot\|_{D_{t}^{(d)}} \rightarrow\|\cdot\|_{\infty}$ as $d \rightarrow \infty$ for all $t \in[0,1]$. Denote by $\boldsymbol{Z}$ and $\hat{\boldsymbol{Z}}^{(d)}$, $d \in \mathbb{N}$, the generator processes of $\boldsymbol{\eta}$ and $\hat{\boldsymbol{\eta}}^{(d)}, d \in \mathbb{N}$. Define

$$
m:=\mathbb{E}\left(\sup _{t \in[0,1]} Z_{t}\right)<\infty, \quad \tilde{Z}:=\frac{\sup _{t \in[0,1]} Z_{t}}{m} .
$$

Then $\mathbb{E}(\tilde{Z})=1$ and, thus, $\left(Z_{t}, \tilde{Z}\right)$ defines a generator for all $t \in[0,1]$. Denote by $\|\cdot\|_{\tilde{D}}$ the $D$-norm pertaining to this generator. Lemma 2 and Corollary 1 imply that $\hat{\boldsymbol{Z}}^{(d)} \leq \boldsymbol{Z}$ for all $d \in \mathbb{N}$. Therefore, for arbitrary $x, y \in \mathbb{R}, d \in \mathbb{N}$, and $t \in[0,1]$, we have

$$
\max \left(|x| Z_{t},|y| \hat{Z}_{t}^{(d)}\right) \leq \max \left(|x| Z_{t},|m y| \tilde{Z}_{t}\right),
$$

where $\mathbb{E}\left(\max \left(|x| Z_{t},|m y| \tilde{Z}_{t}\right)\right)=\|(x, m y)\|_{\tilde{D}}<\infty$. Hence, the dominated convergence theorem, together with the fact that $\hat{Z}_{t}^{(d)} \rightarrow Z_{t}$ as $d \rightarrow \infty$ for all $t \in[0,1]$ by Theorem 1 , implies that

$$
\|(x, y)\|_{D_{t}^{(d)}}=\mathbb{E}\left(\max \left(|x| Z_{t},|y| \hat{Z}_{t}^{(d)}\right)\right) \longrightarrow \mathbb{E}\left(\max \left(|x| Z_{t},|y| Z_{t}\right)\right)=\|(x, y)\|_{\infty}
$$

as $d \rightarrow \infty$ for all $x, y \in \mathbb{R}$.

In Example 1 we have already calculated $\int_{0}^{\infty}\|(1, u)\|_{\infty}^{-2} \mathrm{~d} u=2$. Since $\|\cdot\|_{\infty}$ is the least $D$-norm, we have for all $d \in \mathbb{N}$ and $t \in[0,1]$,

$$
\frac{1}{\|(1, u)\|_{D_{t}^{(d)}}^{2}} \leq \frac{1}{\|(1, u)\|_{\infty}^{2}}
$$

and, therefore, again by the dominated convergence theorem

$$
\int_{0}^{\infty} \frac{1}{\|(1, u)\|_{D_{t}^{(d)}}^{2}} \mathrm{~d} u \longrightarrow \int_{0}^{\infty} \frac{1}{\|(1, u)\|_{\infty}^{2}} \mathrm{~d} u=2 \quad \text { as } d \rightarrow \infty
$$

which completes the proof.

\section{Extensions and future work}

\subsection{Reconstruction of a standard generalized Pareto process}

In this section we apply the generalized max-linear model to generalized Pareto processes (GPPs). Since most of the results of the previous sections carry over very straightforwardly, we only give a short summary of the statements. For a detailed review; see Falk et al. (2014). 
We call a stochastic process $\boldsymbol{V} \in \bar{C}^{-}[0,1]$ a standard generalized Pareto process (SGPP) if there exists a $D$-norm $\|\cdot\|_{D}$ on $E[0,1]$ and some $c>0$ such that $\mathbb{P}(\boldsymbol{V} \leq f)=1-\|f\|_{D}$ for all $f \in \bar{E}^{-}[0,1]$ with $\|f\|_{\infty} \leq c$. Note that this implies that each univariate marginal distribution of $\boldsymbol{V}$ coincides in the upper tail with the uniform distribution on $[-1,0]$. For a detailed examination of GPPs; see Ferreira and De Haan (2014).

Just like in the SMSP case, we can use the generalized max-linear model to discretize and reconstruct a given SGPP. Let $\boldsymbol{V}=\left(V_{t}\right)_{t \in[0,1]}$ be an SGPP with generator process $\boldsymbol{Z}=$ $\left(Z_{t}\right)_{t \in[0,1]}$ and $D$-norm $\|\cdot\|_{D}$. Choose a grid $0=: s_{0}<s_{1}<\cdots<s_{d-1}<s_{d}:=1$ of points within $[0,1]$. Then $\left(V_{s_{0}}, \ldots, V_{s_{d}}\right)$ is a standard GPD RV in $\mathbb{R}^{d+1}$ with pertaining $D$-norm $\|\cdot\|_{D_{0, \ldots, d}}$ generated by $\left(Z_{s_{0}}, \ldots, Z_{s_{d}}\right)$, i.e. there is a vector $\boldsymbol{y}^{(0)}=\left(y_{0}^{(0)}, \ldots, y_{d}^{(0)}\right)<\mathbf{0}$ such that $\mathbb{P}\left(V_{s_{0}} \leq y_{0}, \ldots, V_{s_{d}} \leq y_{d}\right)=1-\|\boldsymbol{y}\|_{D_{0, \ldots, d}}$ for all $\boldsymbol{y}=\left(y_{0}, \ldots, y_{d}\right)$ with $\boldsymbol{y}^{(0)} \leq \boldsymbol{y} \leq \mathbf{0}$. For a detailed examination of GPD RV; see, e.g. Falk et al. (2011).

We apply the generalized max-linear model to the GPD RV $\left(V_{s_{0}}, \ldots, V_{s_{d}}\right)$ and obtain a stochastic process $\hat{\boldsymbol{V}}=\left(\hat{V}_{t}\right)_{t \in[0,1]}$,

$$
\hat{V}_{t}:=\max _{i=0, \ldots, d} \frac{V_{s_{i}}}{g_{i}(t)},
$$

where $g_{0}, \ldots, g_{d} \in \bar{C}^{+}[0,1]$ are functions satisfying (1). It is easy to show that this process defines an SGPP with the generator process $\hat{Z}=\left(\hat{Z}_{t}\right)_{t \in[0,1]}$,

$$
\hat{Z}_{t}=\max _{i=0, \ldots, d}\left(g_{i}(t) Z_{s_{i}}\right), \quad t \in[0,1]
$$

Therefore, for all $f \in \bar{E}^{-}[0,1]$ close enough to 0 , we have

$$
\mathbb{P}(\hat{\boldsymbol{V}} \leq f)=1-E\left(\sup _{t \in[0,1]}\left(|f(t)| \hat{Z}_{t}\right)\right) .
$$

By choosing the exact same functions $g_{0}^{*}, \ldots, g_{d}^{*}$ as in the special case of the generalized max-linear model in Section 3, we obtain the SGPP,

$$
\begin{aligned}
\hat{V}_{t} & =\max \left(\frac{V_{s_{i-1}}}{g_{i-1}^{*}(t)}, \frac{V_{s_{i}}}{g_{i}^{*}(t)}\right) \\
& =\left\|\left(s_{i}-t, t-s_{i-1}\right)\right\|_{D_{i-1, i}} \max \left(\frac{V_{s_{i-1}}}{s_{i}-t}, \frac{V_{s_{i}}}{t-s_{i-1}}\right), \quad t \in\left[s_{i-1}, s_{i}\right], i=1, \ldots, d .
\end{aligned}
$$

This process interpolates the GPD RV $\left(V_{0}, \ldots, V_{d}\right)$. In complete accordance to the SMSP case, we call the process $\hat{\boldsymbol{V}}$ from (6) the discretized version of $\boldsymbol{V}$ with grid $\left\{s_{0}, \ldots, s_{d}\right\}$. Clearly, the pathwise structure of the discretized version of an SMSP that we established in Lemma 3 carries over to the SGPP case.

Now consider a sequence of discretized versions $\hat{\boldsymbol{V}}^{(d)}$ of an SGPP $\boldsymbol{V}$ with grid $g_{d}$ where the diameter of $g_{d}$ converges to 0 . Repeating the arguments in the proof of Theorem 1 yields the pathwise uniform convergence of this sequence to $V$ with probability 1 .

It is also possible to calculate some kind of mean-squared error of the predictor $\hat{V}_{t}$ of $V_{t}$, but in contrast to the case of max-stable distributions, we typically only know the distribution of a GPD RV in the upper tail. This fact forces us to deal with conditional expectations that lead to quite tedious calculations. We state only the main result here. For more more details, see Falk et al. (2014). 
Theorem 3. Let $\boldsymbol{V}$ and $\hat{\boldsymbol{V}}^{(d)}, d \in \mathbb{N}$, be as above. Suppose that $\|\cdot\|_{D_{t}^{(d)}} \neq\|\cdot\|_{1}, d \in \mathbb{N}$, where $\|\cdot\|_{D_{t}^{(d)}}$ is the D-norm pertaining to $\left(V_{t}, \hat{V}_{t}^{d}\right)$. Then for c close enough to 0 , we have

$$
\mathbb{E}\left(\left(V_{t}-\hat{V}_{t}^{(d)}\right)^{2} \mid V_{t}>c, \hat{V}_{t}^{(d)}>c\right) \longrightarrow 0 \text { as } d \rightarrow \infty .
$$

Note that the bivariate GPD $W(x, y)=1-\|(x, y)\|_{1}$ has to be treated with caution. It represents the case of uniform distribution on the line $\{(x, y): x, y \leq 0, x+y=-1\}$, which means that no observations fall in any rectangle $[u, 0] \times[v, 0], u+v \geq-1$ (cf. Falk et al. (2011)).

\subsection{Generalized max-linear models in an arbitrary dimension}

Suppose now that $\boldsymbol{\eta}=\left(\eta_{\boldsymbol{t}}\right)_{\boldsymbol{t} \in[0,1]^{m}}$ is an SMSP on $[0,1]^{m}$, which we observe only through the grid of (different) indices $s_{1}, \ldots, s_{d}$. Denote by $\|\cdot\|_{D_{s_{1}, \ldots, s_{d}}}$ the $D$-norm pertaining to $\left(\eta_{\boldsymbol{s}_{1}}, \ldots, \eta_{\boldsymbol{s}_{d}}\right)$. Choose an arbitrary norm $\|\cdot\|$ on $\mathbb{R}^{d}$ and define for $i=1, \ldots, d$. and $h>0$,

$$
g_{i, h}^{*}(\boldsymbol{t}):=\frac{K\left(\left\|\boldsymbol{t}-\boldsymbol{s}_{i}\right\| / h\right)}{\left\|\left(K\left(\left\|\boldsymbol{t}-\boldsymbol{s}_{1}\right\| / h\right), \ldots, K\left(\left\|\boldsymbol{t}-\boldsymbol{s}_{d}\right\| / h\right)\right)\right\|_{D_{s_{1}, \ldots, s_{d}}}} \quad \text { for } \boldsymbol{t} \in[0,1]^{m},
$$

where $K:[0, \infty) \rightarrow[0,1]$ is a continuous and strictly monotonically decreasing function (kernel) with the following two properties:

$$
K(0)=1, \quad \lim _{t \rightarrow \infty} \frac{K(x t)}{K(y t)}=0, \quad 0 \leq y<x .
$$

The exponential kernel $K_{\mathrm{e}}(t)=\exp (-t), t \geq 0$, is a typical example. Put

$$
\tilde{\eta}_{\boldsymbol{t}, h}:=\max _{i=1, \ldots, d} \frac{\eta_{\boldsymbol{s}_{i}}}{g_{i, h}^{*}(\boldsymbol{t})} \quad \text { for } \boldsymbol{t} \in[0,1]^{m}, h>0 .
$$

It can be shown (cf. Falk et al. (2014)) that $\tilde{\eta}=\left(\tilde{\eta}_{t, h}\right)_{t \in[0,1]^{m}}$ is an SMSP in $C\left([0,1]^{m}\right)$ since the continuity of $K$ implies the continuity of the paths of $\tilde{\eta}$ and each finite-dimensional marginal distribution of $\tilde{\boldsymbol{\eta}}$ is standard max-stable. Furthermore, in this case,

$$
\tilde{\eta}_{s_{j}, h} \longrightarrow \eta_{s_{j}}, \quad h \downarrow 0,1 \leq j \leq d .
$$

Further details of this approximation will be developed in a forthcoming paper.

\section{Acknowledgements}

The authors are grateful to the reviewers of the first version of this paper for their careful reading. The paper has benefited a lot from their constructive remarks. The hints to a possible extension to an arbitrary dimension initiated ongoing research.

\section{References}

Aulbach, S., Falk, M. and Hofmann, M. (2013). On max-stable processes and the functional D-norm. Extremes 16, 255-283.

Buishand, T. A., De HaAn, L. And Zhou, C. (2008). On spatial extremes: with application to a rainfall problem. Ann. Appl. Statist. 2, 624-642.

Davis, R. A. AND Mikosch, T. (2008). Extremal value theory for space-time processes with heavy-tailed distributions. Stoch. Process. Appl. 118, 560-584.

De HaAn, L. (1984). A spectral representation for max-stable processes. Ann. Prob. 12, 1194-1204. 
De HaAn, L. and Ferreira, A. (2006). Extreme Value Theory: An Introduction. Springer, New York. Available at http://people.few.eur.nl/ldehaan/EVTbook.correction.pdf and http://home.isa.utl.pt/ anafh/corrections.pdf for corrections and extensions.

De HaAn, L. And Lin, T. (2001). On convergence toward an extreme value distribution in C[0, 1]. Ann. Prob. 29, 467-483.

De HaAn, L. AND Resnick, S. I. (1977). Limit theory for multivariate sample extremes. Z. Wahrscheinlichkeitsth. 40, 317-337.

Dombry, C., Éyi-Minko, F. And Ribatet, M. (2013). Conditional simulation of max-stable processes. Biometrika 100, 111-124.

Falk, M., Hofmann, M. And Zott, M. (2014). On generalized max-linear models and their statistical interpolation. Preprint. Available at http://arxiv.org/abs/1303.2602v2.

FALK, M., HüsLER, J. AND Reiss, R.-D. (2011). Laws of Small Numbers: Extremes and Rare Events, 3rd edn. Springer, Basel.

Ferreira, A. ANd De HaAn, L. (2014). The generalized Pareto process; with a view towards application and simulation. Bernoulli 20, 1717-1737.

Giné, E., Hahn, M. and Vatan, P. (1990). Max-infinitely divisible and max-stable sample continuous processes. Prob. Theory Relat. Fields 87, 139-165.

Hult, H. AND LindsKog, F. (2005). Extremal behavior of regularly varying stochastic processes. Stoch. Process. Appl. 115, 249-274.

Hult, H. And Lindskog, F. (2006). Regular variation for measures on metric spaces. Publ. Inst. Math. (Beograd) (N. S.) 80, 121-140.

KABLUCHKo, Z. (2009). Spectral representations of sum- and max-stable processes. Extremes 12, 401-424.

PICKANDS, J., III (1981). Multivariate extreme value distributions. Bull. Inst. Internat. Statist. 49, 859-878, 894-902.

Sмith, R. L. (1990). Max-stable processes and spatial extremes. Preprint, University of Surrey. Available at http://www.stat.unc.edu/faculty/rs/papers/RLS_Papers.html.

Stoev, S. A. And Taqqu, M. S. (2005). Extremal stochastic integrals: a parallel between max-stable processes and $\alpha$-stable processes. Extremes 8, 237-266.

Wang, Y. And Stoev, S. A. (2010). On the structure and representations of max-stable processes. Adv. Appl. Prob. 42, 855-877.

Wang, Y. And Stoev, S. A. (2011). Conditional sampling for spectrally discrete max-stable random fields. Adv. Appl. Prob. 43, 461-483. 